\title{
Development of Atom Probe Tomography for Biological Materials
}

\author{
Mark E. Greene,* Ty J. Prosa,* John A. Panitz,** David J. Larson,** and \\ Thomas F. Kelly* \\ *Imago Scientific Instruments Corporation, 5500 Nobel Dr, Madison, WI 53711 \\ ** High Field Consultants, http://highfieldconsultants.com
}

Compositional mapping of biological materials at the atomic scale using atom probe tomography (APT) promises the unprecedented ability to image the chemical structure of single biological molecules in three dimensions (3-D) in real space. Under proper experimental conditions, it is anticipated that individual atoms and molecular fragments from biological and organic macromolecules can be detected such that the time-of-flight data can be spatially reconstructed to provide the 3-D structure of the molecules. To effectively analyze biological and organic specimens using APT, several experimental parameters - such as the choice of substrate, electric field strength, temperature, and laser pulse characteristics - must be optimized to yield mass fragments that can be repeatably evaporated and reliably identified from the typical organic background.

Recent efforts on ferritin have aimed at developing specimen preparation techniques at ambient temperatures, verifying these techniques with transmission electron microscopy (TEM), and gathering initial APT data. Ferritin is a ubiquitous protein responsible for storing iron and is composed of a polypeptide shell with an iron-rich core. Ferritin was chosen for three reasons. First, it is a well-studied and well-characterized protein with significant scientific literature familiar to the biological community. Second, the iron core provides excellent contrast in TEM such that deposition of molecules can be readily verified. Panitz and coworkers used ferritin effectively in this manner [1-4]. Third, the iron should provide a noncontroversial signature in the mass spectrum during atom probe analysis, indicating the presence of ferritin, and should be spatially positioned according to the known structure.

Two primary materials have been investigated as substrates for ferritin molecules: pure tungsten and pure gold. TEM indicates that protein molecules have adsorbed on substrates made from electrochemically sharpened $\mathrm{W}$ and Au wire. The best results were obtained for tips electropolished from Au TEM grids, Fig. 1a, which will be the focus here. Atom probe specimen preparation was multiplexed by electrochemically sharpening 200 mesh $(\approx 100 \mu \mathrm{m}$ pitch) gold TEM grids that were clipped in half. Ferritin layers were deposited from solution onto a row of a dozen sharpened tips simultaneously and verified by TEM, Figs. $1 \mathrm{~b}$ and $1 \mathrm{c}$.

The central tip in the row was analyzed in the atom probe to observe the field evaporation characteristics of the deposited biomaterial as a function of voltage applied to the TEM grid. Deposited material evaporated from the apex up to $6.8 \mathrm{kV}$ at which point the field was terminated. Subsequent TEM imaging indicated that the layer had been evaporated by the electric field, Fig. 1d. Equally importantly, TEM showed that the neighboring tips, Fig. 1e, retained the ferritin deposits after the central tip was exposed to $6.8 \mathrm{kV}$, 
indicating that the electric field is not strong enough at the apex of the other tips to evaporate significant deposited biomaterial. This capability improves specimen throughput by allowing multiple biological and organic samples to be run on a monolithic conducting substrate using laser-assisted local electrode atom probe.

\section{References}

[1] J. A. Panitz and I. Giaever, Surf. Sci. 97 (1980) 25-42.

[2] J. A. Panitz and I. Giaever, Ultramicroscopy 6 (1981) 3-6.

[3] J. A. Panitz, Ultramicroscopy 7 (1982) 241-248.

[4] J. A. Panitz, Rev. Sci. Instrum. 56 (1985) 572-574.

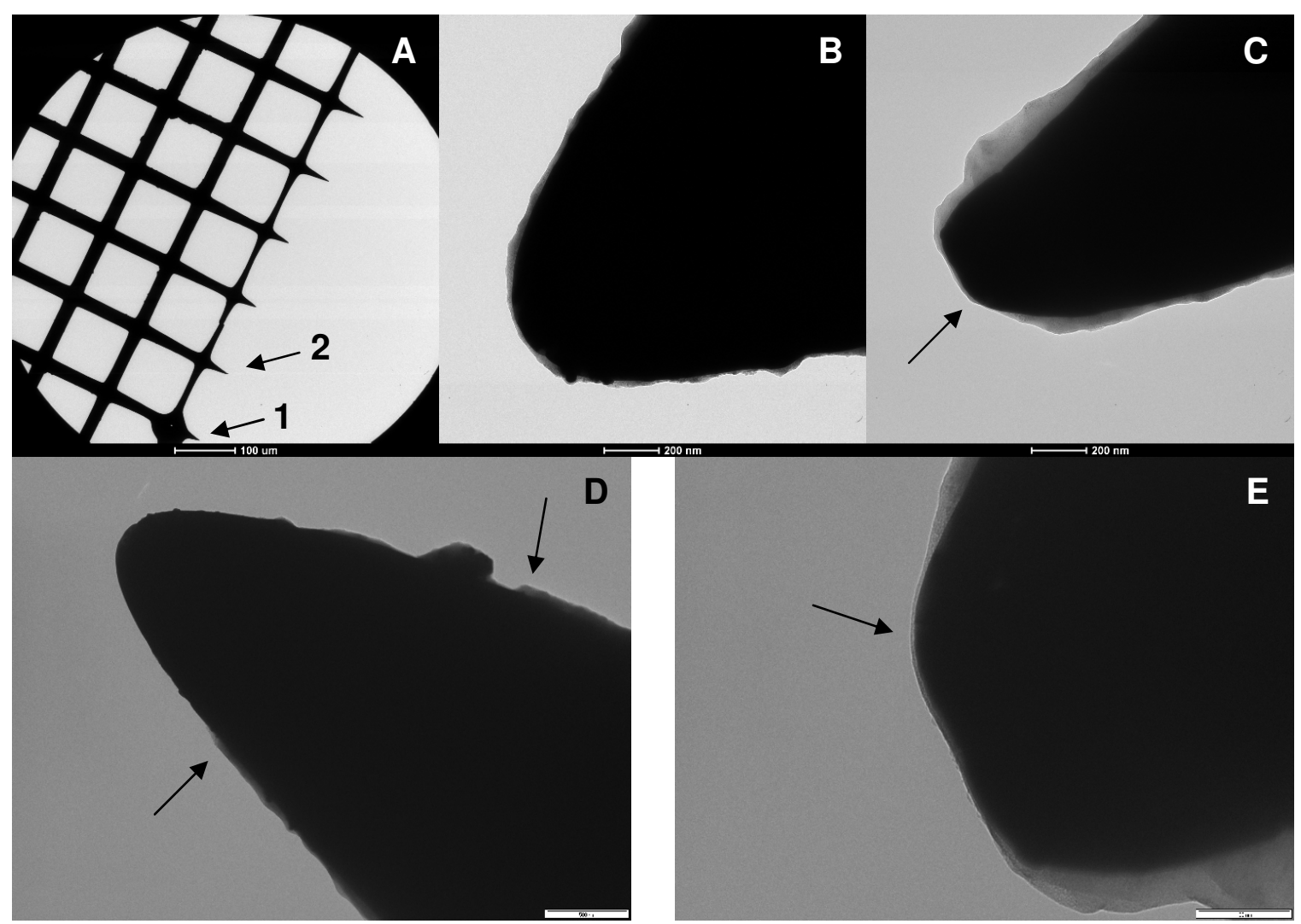

Fig. 1. TEM of Au tips covered in ferritin before and after atom probe analysis. (A) Row of Au tips from an electrochemically sharpened TEM grid. Tip 1 was analyzed in the atom probe. Tip 2 was a neighboring tip not analyzed by atom probe. Scale bar: $100 \mu \mathrm{m}$. (B) Tip 1 covered with a layer of ferritin deposited from solution. Scale bar: $200 \mathrm{~nm}$. (C) Tip 2 also covered with a layer of ferritin deposited from solution. Arrow indicates area at apex with thin layer of ferritin. Scale bar: $200 \mathrm{~nm}$. (D) Tip 1 after atom probe analysis up to $6.8 \mathrm{kV}$ showing that the ferritin layer has been removed at the apex by the electric field. The remaining layer can be seen about a micron down the shank, indicated by the arrows. Note image in $\mathrm{D}$ is flipped vertically with respect to image in B. Scale bar: 500 $\mathrm{nm}$. (E) Tip 2 after atom probe analysis of tip 1 showing that the ferritin layer remains intact. Arrow indicates area of thin layer noted in image C. Note image in E is flipped vertically with respect to image in C. Scale bar: $100 \mathrm{~nm}$. 